\title{
Vascularization in interconnected 3D printed Ti-6Al- 4V foams with hydrogel matrix for biomedical bone replacement implants
}

\author{
Victor L. Correa ${ }^{1 *}$, Kristine M. Garza ${ }^{2}$ and Lawrence E. Murr ${ }^{1}$
}

\begin{abstract}
Vascularization on newly implanted metal orthopedic implants has remained a challenge in the field of tissue engineering. To address this challenge, in this research, an interconnected foam structure of Ti-6Al-4V was microfabricated by electron beam melting (EBM) technique. The foam in question has a density of $1.77 \mathrm{~g} \mathrm{~cm}^{-3}$ with $60 \%$ porosity and a tensile strength of $18 \mathrm{GPa}$. An extracellular matrix based hydrogel was added as an aqueous matrix to the foam. Hypoxia mimetic stress has been closely related to many wound healing biomedical applications as it increases survival and proliferation molecular signals. To that end, increased expression of hypoxia-inducible factor-1 $\alpha$ (Hif-1 $\alpha$ ) and vascular endothelial growth factor (VEGF) in the aqueous hydrogel matrix was achieved by the addition of a hypoxia mimetic deferoxamine mesylate (DFM). In this study, the formation of an endothelial network was achieved in a hydrogel matrix in the presence of the before mentioned 3D printed metal foam. Cellular viability, fluorescent microscopy and scanning electron microscopy imaging analysis demonstrate that pre-osteoblasts undergo proliferation and also attach efficiently to the foam when exposed to DFM. Human umbilical vascular endothelial cells (HUVECs) were grown in an extracellular matrix-like 3D hydrogel and a hypoxia-like stress was achieved. This research demonstrates that pre-osteoblast cells undergo cell differentiation and increase the production of hydroxyapatite on exposure to the hypoxia mimetic molecule. This proposed approach encompasses an ideal prototype for a completely living implanted structure for future orthopedic implants.
\end{abstract}

Keywords: Ti-6Al-4V, electron beam melting, angiogenesis, deferoxamine mesylate, hydrogel, vascularization

\section{INTRODUCTION}

3D printing or additive manufacturing (AM) has revolutionized the way materials scientists and engineers synthesize and manufacture materials. This manufacturing technology allows bioengineers to enhance metals, composites, polymer plastics, and, in the context of this research: biomedical devices. Tissue engineering is an emerging field, in which materials that display biomimetic properties are employed for medical applications. Despite the considerable amount of research advances being made [1-5], the main challenge in tissue engineering is to create a fully functional living system parting from a non-living scaffold. There exists an ever present need to develop materials that are not just biocompatible, but that can more closely mimic a complete biological system. Advances in the integration of implants allows for the regeneration of lost tissue. Enhancing the integration of implants has a wide variety of applications that can reduce medical costs while improving an individual's quality of life. Applications include controllable prosthetics, nerve and muscle regeneration, and improved healing of bone fractures in patients with diseases such as diabetes [6].

Bone is composed mainly of mineralized calcium crystals (hydroxyapatite), with a chemical formula of $\mathrm{Ca}_{5}$ $\left(\mathrm{PO}_{4}\right)_{3}(\mathrm{OH})$, and collagen. The mixture of these materials provides mechanical support and a degree of elasticity required for bone tissue. Despite the success of implanted orthopedics, there has been piling evidence of infection, aseptic loosening, and pain without loosening or other reasons of failure [7]. Cells at the interface between the bone and the implant cannot grow into the solid bio-

\footnotetext{
${ }^{1}$ Department of Metallurgical, Materials and Biomedical Engineering, University of Texas at El Paso, El Paso, TX 79902, USA

2 Department of Biological Sciences, University of Texas at El Paso, El Paso, TX 79902, USA

* Corresponding author (email: vlcorrearodriguez@utep.edu)
} 
compatible metal alloy. To this day, bone replacement implants remain as solid structures, providing a physical barrier for cells to grow. This limits the ingrowth of bone into the implant, and consequently results in a lack of regeneration of tissue.

Many strategies have been employed in order to enhance the bio-compatibility of orthopedic implants $[2,4,8-11]$, but a more diverse set of materials need to be examined in order to closely mimic a complete living system. To this end, 3D printed Ti-6Al-4V foams are an effective alternative, given their degree of porosity. Advantages to this approach include varying pore size gradient, varying porosity, and a high degree of resolution control on the implant synthesis. An extracellular matrixlike gel in combination with 3D printed Ti-6Al-4V foams was evaluated for the development of a bone replacement implant. In this research, a Ti-6Al-4V foam structure was designed to promote cell migration of vascular endothelial cells and pericytes, and differentiation and proliferation of pre-osteoblast cells. Given that there is a degree of porosity in these foams, a matrix can be applied to the structure, allowing for microcapillar formation in a 3D suspension. Since hydrogels are highly hydrated polymers, certain molecules and growth factors can be mixed into it, providing cells with the necessary supplements required for proliferation and growth. This research focuses on determining the ability of the before mentioned materials to drive vascularization, and enhance bone repair, while maintaining mechanical and structural similarities with real bone.

The process of the development of new vasculature (angiogenesis) is of vital importance in the process of wound healing [12]. Vascular structures serve as transport pathways for oxygen, nutrients and signaling molecules throughout the organismal systems. Because of the significance of this process, the capacity of implant to induce vascularization is essential to develop an ideal substitute of the original biological matter. The main role of an endothelium is to serve as a transport pathway for oxygen. Therefore, endothelial cells are equipped with oxygen sensor proteins such as prolyl hydroxylase domain enzymes (PHDs) which down-regulate the levels of hypoxia inducible factors-1a (Hif- $1 \alpha$ ). Despite the biological importance of vascular structure formation, achieving vascular network maturation remains the main challenge when engineering tissue [13].

Cells have evolved to respond to varied environments. Lack of free oxygen is one of them. Because oxygen is required for many cellular metabolic processes, such as the production of adenosine triphosphate (ATP), fatty acid synthesis and oxidative phosphorylation, cells are prepared to activate transcription factors that promote cell survival [14]. VEGF administration to cells grown in 3D cell cultures has been reported to promote an angiogenic response, and increase the activation of the phosphatidyl inositol-3-kinase (PI3K)-Akt signaling pathway [15]. Hypoxia mimetics have been reported to increase the viability of cells and progression of survival signaling pathways $[15,16]$. On a normal cell line, inhibiting the degradation of Hif- $1 \alpha$ inhibits apoptosis, does not produce reactive oxygen species (ROS), but results in promoting cellular differentiation and migration. The PI3K-Akt pathway becomes activated while a cell is experiencing a hypoxic response. Deferoxamine mesylate (DFM) is an iron chelating agent, meaning that it binds to free iron ions in solution. This molecule is employed to regulate iron homeostasis in cells by chelating iron in solution $[17,18]$. DFM is a well known inhibitor of PHD enzymes and has also been shown to increase bone density in osteoporosis mouse models [19]. PHD enzymes require iron co-factors in order to add hydroxyl groups to proline residues. Because DFM binds to iron co-factors, the catalytic ability of PHD enzymes becomes hindered, leading to the intracellular stabilization of Hif1a. DFM has been approved by the Food and Drug Administration (FDA) and is available for clinical use in the US. Currently, it is being used as an iron chelating agent to treat iron overdose from blood transfusions [20]. Iron chelation by DFM administration has also shown that bone resorption is inhibited by limiting osteoclastic differentiation $[21,22]$.

A number of research papers have been published with promising applications for hypoxia in wound healing $[6,23]$ in recent years. These approaches include but are not limited to diabetic wound healing in fibroblasts [6], several mitochondrial related metabolic diseases such as Leigh syndrome [24], and more recently to treat brain hemorrhage [25]. The biomedical applications of hypoxia can be tailored to combat a variety of wound healing situations. It has been reported that inhibiting PHD-2 enzymes and stabilizing Hif- $1 \alpha$ increase the survival rate of newly implanted cells in bone [23]. De-activation of PHD-2 has resulted in a decrease on the levels of ROS species in endothelial cells, enabling cells to undergo redox homeostasis and glycogen storage [23]. This further suggests that a hypoxia mimetic, but not hypoxia as a lack of oxygen maintains the integrity of cellular metabolism by stabilizing glutathione $S$ transferase (GST). Because of the ever increasing evidence that hypoxia can support regenerative medicine, in this research, a hypoxia mi- 
metic will be applied to promote vascularization, preosteoblast differentiation and wound healing for newly implanted bone replacement implants.

The ingrowth of bone into the implant is essential in order to achieve what is conceptually a "living implant". Although the main goal of this research is to stimulate the formation of vascular structures within the porous metal implant, the nature of wound healing must also be addressed. This includes, but is not limited to the mineralization of calcium by osteoblasts, the inhibition of bone resorption by osteoclasts, and avoiding debris release by the metal. Ideally, a bone replacement implant should promote osteoblast differentiation to avoid stress shielding and promote tissue regeneration. Another challenge to tackle in designing a living implant is the release of potentially toxic metal debris which can be attributed to mechanical loading on the material. In several cases, Ti$6 \mathrm{Al}-4 \mathrm{~V}$ particulate debris has been found in oral mucosa of patients with dental implants of this material [26].

The main goal of this research is to design a bone replacement implant capable of forming vascular structures in a hydrogel matrix, while allowing for osteoblast proliferation and cell differentiation. Osteoblasts can also successfully synthesize hydroxyapatite and retain their adhesion to the Ti-6Al-4V foam [27]. The hydrogel matrix should contain all of the necessary supplements to favor angiogenesis and vascular structure maturation. The process of wound healing includes the re-establishing of osteoblast mediated synthesis of calcium oxalate, the ability of endothelial cells to form a microvasculature and ensuring the maturity and stability of said vasculature. It is expected that bone tissue will grow into the foam structure. The implant design is porous and contains a matrix that stimulates the ingrowth of bone tissue. Stress shielding is one of the leading causes of bone replacement implant failure. This type of failure is driven by osteoclasts, and it occurs when the rate of bone resorption is higher than the rate of bone formation [28]. Ultimately, this approach is expected to produce what will become a prototype of a living implant-an engineered mixture of materials that will work to orchestrate appropriately all of the biological processes of real bone.

\section{EXPERIMENTAL SECTION}

\section{Material fabrication, preparation and characterization}

An interconnected foam structure of Ti-6Al-4V was micro-fabricated by electron beam melting (EBM) technique. The spherical powder size that was used was in the range of $30 \mu \mathrm{m}$ in diameter. We used an ARCAM-EBM system for the fabrication of foam structures. The density was determined by using the Archimedes Principle for sintered powder metallurgy products ASTM B962-13.

The foam structures fabricated via the EBM system were qualitatively characterized by scanning electron microscopy (SEM) and quantitatively through a densitometry approach. The average pore size was estimated from the SEM micrographs, whereas, porosity and modulus were calculated from densitometric measurements using the following equation:

$$
\text { Porosity }(\%)=\left[1-\frac{\rho}{\rho_{0}}\right] \times 100 \%,
$$

and Gibson-Ashby equation:

$$
\frac{E}{E_{0}}=\left(\frac{\rho}{\rho_{0}}\right)^{n},
$$

where $E$ and $E_{0}$ are the stiffness for an open-cellular structure and solid (fully dense) material having a density of $\rho$ and $\rho_{0}$, respectively. For Ti-6Al-4V alloy, $E_{0}=$ $110 \mathrm{GPa}, \rho_{0}=4.43 \mathrm{~g} \mathrm{~cm}^{-3}, n$ varies from $\sim 1.8-2.2$, and can be approximated to be 2 . These values were determined in prior work [29].

\section{Implementation of a hydrogel matrix on the Ti-6Al-4V foam}

In order to produce a fully functional orthopedic implant, an aqueous matrix needs to be added to the printed foam structures. Cells may grow freely in this structure so they may behave as they would in normal tissue. The cell biology of angiogenesis is a tightly regulated and complex process, orchestrated only when necessary. A myriad of cell types, such as endothelial cells, pericytes, osteoblasts, etc. have roles to fulfill in the process of wound healing in bone, all of which need to interact with each other, differentiate and migrate in 3D tissue for complete wound healing.

Given that there is such a high level of cellular organization in the process of forming new vasculature, the aqueous matrix employed in this research needs to be as similar to an extra cellular matrix (ECM) as possible. To this end, Corning ${ }^{\oplus}$ Matrigel ${ }^{\circ}$ Matrix was evaluated as a potential matrix, given that its main constituents are ECM proteins such as laminin, collagen IV, heparin sulfate proteoglycans, entactin/nidogen, and a number of growth factors. A thin section of Ti-6Al-4V foam was placed in a 12 well tissue culture plate. Corning ${ }^{\otimes}$ Matrigel $^{\circ}$ Matrix was added in each well containing the foam until fully covered. 


\section{Cell culture}

The MC3T3-E1 subclone 4 (mouse pre-osteoblasts (ATCC ${ }^{\bullet}$ Manassas, VA, USA CRL -2593 ${ }^{\text {TM}}$ )) cell line was used as a pre-osteoblast model for this research. MC3T3E1 subclone 4 were grown in alpha minimum essential medium ( $\alpha$-MEM, Sigma-Aldrich) cell medium supplemented with $10 \%$ fetal bovine serum (FBS, ATCC 302020).

Human umbilical vein endothelial cells (HUVECs, ATCC $^{\circ}$ Manassas, VA, USA CRL-1730 ${ }^{\mathrm{TM}}$ ) were grown in 1:1 F-12K/DMEM media supplemented with $10 \% \mathrm{FBS}$, $0.1 \mathrm{mg} \mathrm{mL}^{-1}$ heparin; $0.03-0.05 \mathrm{mg} \mathrm{mL}^{-1}$ endothelial cell growth supplement (ECGS, Sigma-Aldrich). Cells were incubated in a $5 \% \mathrm{CO}_{2}$ environment at $37^{\circ} \mathrm{C}$.

Cell media was changed every two days and washed with $1 \times$ PBS solution with every media change for the MC3T3-E1 cells. HUVEC cell media was aspirated and centrifuged at $1,500 \mathrm{rpm}$ for $5 \mathrm{~min}$ in order to collect cellular debris that is essential for their proliferation. Cells are sub-divided by trypsin method. Cells were incubated with $0.25 \%$ trypsin/ethylene diamine tetraacetic acid (EDTA) solution for 5-7 min until cells are no longer attached to the bottom of the culture vessel, stained with trypan blue exclusion dye and counted using a hemocytometer.

\section{Seeding efficiency}

Cellular Ti-6Al-4V alloy foam structures and metallographically polished flat Ti-6Al-4V alloy samples were seeded with pre-osteoblasts $(200,000$ cells/well in a 12 well cell culture plate) and incubated for $12 \mathrm{~h}$ at $37^{\circ} \mathrm{C}$ in a $\mathrm{CO}_{2}$ incubator to examine cell seeding efficiency. After incubating for $12 \mathrm{~h}$, the specimens were removed from their respective wells with a trypsin solution and the cells remaining in each well were stained with trypan blue and counted using hemocytometer. The number of live cells estimated from the hemocytometer was subtracted from the total number of initially seeded cells, to obtain the number of cells that were seeded on each specimen. The seeding efficiency value was calculated as described in the following equation:

Seeding efficiency $(\%)=$

$\left(\frac{(\text { Total number of cells seeded })-(\text { Cells attached to the well })}{\text { Total number of cells seeded }}\right)$ $\times 100$.

\section{MTS viability assay protocol}

To study lethal effect that DFM may have on MC3T3-E1 and HUVEC cells, an 3-(4,5-dimethylthiazol-2-yl)-5-(3carboxymethoxyphenyl)-2-(4-sulfophenyl)-2H-tetra- zolium (MTS) viability assay (Promega) was performed. Hypoxia itself has not been reported to be cytotoxic to cells; however, some compounds may trigger a hypoxic response and produce a cytotoxic response, such as $\mathrm{CoCl}_{2}$. In this experiment, the viability of both pre-osteoblasts and endothelial HUVEC cells were compared with exposures of 1:5 dilutions of DFM and $\mathrm{CoCl}_{2}$ as a positive toxic response at various time exposures $(24 \mathrm{~h}$, $48 \mathrm{~h}, 72 \mathrm{~h}, 5$ day and 14 day). In order to determine which concentrations of DFM are toxic to both MC3T3E1 cells and HUVECs, an initial test was performed. This consisted in seeding 50,000 cells/well in a total volume of $200 \mu \mathrm{L}$ of media with the 1:5 dilutions ranging from 2 $\mathrm{mmol} \mathrm{L}^{-1}$ to $640 \mathrm{nmol} \mathrm{L}^{-1}$ of each compound. The starting stock solution of both hypoxia inducers was 10 $\mathrm{mmol} \mathrm{L}^{-1}$. Cell media was changed every three days during the time of the experiment to avoid starvation related issues.

For cells grown in 3D printed foams, pre-osteoblast cells were seeded as previously described at a density of 200,000 cells/well in 12 well cell culture plates containing the $3 \mathrm{D}$ printed Ti-6Al-4V scaffold disks for the previously specified amount of time and dilutions of DFM. For this experiment, a negative control consisted of cells growing of the tissue culture plate. After the desired periods of incubation, the scaffold was removed from the well with the cells attached to it to a new plate. Trypsinizing cells growing on foam samples has previously proven to produce inconsistent results and also requires extended trypsin incubation, leading to cellular detriment that may alter the viability results. It is for this reason that viability was measured without having to remove the cells from the foams by using the Vybrant ${ }^{\oplus}$ CFDA SE Cell Tracer Kit (Invitrogen). Fresh complete $\alpha$-MEM media was added to the foams with cells. Subsequently, each foam was treated with $1 \mu \mathrm{mol} \mathrm{L}^{-1}$ of Vybrant ${ }^{\circ}$ CFDA SE reagent A dissolved in reagent $B$, as indicated by the kit's specifications.

To perform the viability assay, $20 \mu \mathrm{L}$ of the MTS stock solution $\left(2 \mathrm{mg} \mathrm{mL}^{-1}\right)$ was added to each well. A negative control of $20 \mu \mathrm{L}$ of the MTS stock solution added to $100 \mu \mathrm{L}$ of medium was included. The 96 well plate was incubated at $37^{\circ} \mathrm{C}$ for $4 \mathrm{~h}$ and read absorbance at $490 \mathrm{~nm}$. All experiments were performed in triplicates for statistical significance.

\section{Cell proliferation analysis}

Cell proliferation was analyzed by counting cells by the trypan blue exclusion dye test. MC3T3-E1 cells were seeded at 50,000 cells/well and exposed to 1:5 dilutions of 
DFM and $\mathrm{CoCl}_{2}$ in $200 \mu \mathrm{L}$ total volume for $24 \mathrm{~h}, 48 \mathrm{~h}$, $72 \mathrm{~h}, 5$ day and 14 day. Cells were then trypsinized, stained and counted. The population of live cells was determined by cell count. Each exposure was performed in statistical triplicates.

\section{Fluorescent microscopy}

Pre-osteoblasts seeded on foam structures were cultured for 7 day to test for similar cell behavior as reported by Nune et al. [27]. Cells were seeded at 200,000 cells/well in a 12 well plate, $1 \mathrm{~mL}$ total media volume and then stained with Hoechst 33342 cell nuclei dye. A second experiment was performed once the appropriate concentration of DFM was selected. In this experiment, pre-osteoblasts were seeded at the previously described concentration in the same conditions but exposed to $3.2 \mu \mathrm{mol} \mathrm{L}{ }^{-1} \mathrm{DFM}$. Proliferation was observed after 21 day exposure. A second stain was performed with Vybrant ${ }^{\circledR}$ CFDA SE Cell Tracer Kit to visually determine the viability of cells without disturbing the layer of cells grown in the foams.

Because the scaffold is solid metal, light cannot be transmitted through it if fully dense. However, due to the foam nature of the scaffold, light may be transmitted through the medium and a visible image can be obtained if the metal section is thin enough. Despite the fact that a thin section makes fluorescent microscopy feasible, since the scaffold is $3 \mathrm{D}$, focus is lost in the $z$ axis and there is a lack of clarity for morphological details. Improvements on microscopy can be done by using confocal microscopy instead of fluorescent microscopy in order to view in more detail cellular morphology and microcapillary formation. Cell nuclei was stained with Hoechst 33342 dye at a working dilution of $1 \mu \mathrm{g} \mathrm{mL}^{-1}$ in complete media and incubated for $1 \mathrm{~h}$. After the incubation period of time, the Ti-6Al-4V scaffold was flipped upside down to better observe the cells that grow on the uppermost section of the scaffold. Microscopy was performed in a Zeiss Axiovert 200 fluorescent microscope.

\section{Induction of angiogenesis in foams containing hypoxia mimetic ECM}

It has been previously reported that MC3T3-E1 pre-osteoblast cells undergo differentiation and proliferation when grown in porous foams in glutamine containing media [28]. It has been extensively reported that endothelial cells are able to form capillaries in gels when exposed to DFM [30]. In this experiment, a live angiogenesis monitoring experiment was performed in order to analyze capillary maturation and cell survival. This analysis is achieved by staining HUVECs with PHK26 red fluorescent dye (Sigma) and MC3T3-E1 cells with PKH67 green fluorescent dye (Sigma). Both dyes stain cell membranes in an unspecific way, while maintaining cellular viability for an extended period of time. MC3T3-E1 cells were grown in foams as previously described in the previous section, but under hypoxic conditions (3.2 $\mu \mathrm{mol} \mathrm{L}{ }^{-1}$ DFM) for a total of 7 day, with a negative hypoxia control being cells without any DFM. The cells grown on the foams and gel were monitored throughout the duration of this experiment though fluorescent microscopy. The foams were removed from the wells and placed in a separate 12 well plate. Matrigel ${ }^{\varpi}$ (Corning Life Sciences) was added until the foam was completely covered. In order to observe microcapillar formation upon exposure of HUVECs to DFM, the manufacturer specifications were followed. Briefly, Matrigel $^{\oplus}$ was thawed according to specifications $\left(4^{\circ} \mathrm{C}\right.$ overnight) and added to a 24 well cell culture plate and allowed to gelate also according to specifications $\left(30-60 \mathrm{~min}\right.$ at $\left.37^{\circ} \mathrm{C}\right)$. HUVECs $(70 \%-80 \%$ confluency) are tripsinized, re-suspended $\left(1.2 \times 10^{5}\right.$ cells in $300 \mu \mathrm{L}$ of complete media $(10 \%$ FBS)) and treated with $10 \mu \mathrm{mol} \mathrm{L}^{-1}$ of DFM. The cell suspension was incubated at $37^{\circ} \mathrm{C}, 5 \% \mathrm{CO}_{2}$ for 16 to $18 \mathrm{~h}$ on the Matrigel coated plate. The formation of microcapillar structures was measured by confocal microscopy.

\section{Cell morphology and adhesion}

SEM was used to study the morphology, pore interconnectivity, and in-growth of pre-osteoblasts upon exposure to DFM. Cells were grown for 7 and 14 days. Cells grown on foam samples without any hypoxic inducing molecules served as negative control for this experiment. The cell-seeded samples were fixed with $2.5 \%$ glutaraldehyde in $0.1 \mathrm{~mol} \mathrm{~L}^{-1}$ cacodylate buffer ( $\mathrm{pH}$ 7.4) for $20 \mathrm{~min}$, rinsed with $1 \times \mathrm{PBS}$, dehydrated with a graded series of ethanol $(25 \%-100 \%)$ and critical point dried. Prior to examination of samples via SEM (Hitachi S$4800)$, the samples were sputter-coated with $10 \mathrm{~nm}$ of palladium to ensure sample conductivity and improve image resolution. The calcium-content in the ECM was estimated after the DFM exposure via energy dispersive $\mathrm{X}$-ray analysis (EDAX) in the SEM.

\section{Alizarin red $S$ (ARS) mineralization assay}

Because it is expected that pre-osteoblasts differentiate into osteoblasts under a hypoxic condition, cells should mineralize calcium in the ECM. The study of mineralization of ECM can be viewed as a measure or potential for bone formation. In this regard, alizarin red assay (ARS) (Sigma-Aldrich) was used to qualitatively and 
quantitatively indicate the presence of calcium in the matrix. Cells were grown on foams at the selected optimum DFM concentration for $48 \mathrm{~h}$. Cells grown in 3D printed foams in complete media with no added glutamate served as the negative control for this experiment. After incubation, the samples were washed carefully with PBS without disturbing the cell layer and fixed with $4 \%$ paraformaldehyde for $30 \mathrm{~min}$. The 3D foam structures were then stained with alizarin red $(\mathrm{pH} 4.1)$ in dark and at room temperature for $30 \mathrm{~min}$. Samples were qualitatively studied via light microscope after rinsing with PBS. The calcium nodules should appear red. To conduct quantitative analysis, $10 \%$ cetyl pyridinium chloride was added to remove the alizarin red stain. The absorbance of the solution was measured at $570 \mathrm{~nm}$ OD with a spectrophotometric microplate reader.

\section{Statistical analysis of data}

The data was normalized with respect to control experiments and expressed as the mean of at least three replicates with standard deviation (SD). Three sets of experiments were carried out for each experimental run.

\section{RESULTS}

\section{Material fabrication, preparation and characterization} Ti-6Al-4V foams were successfully synthesized and characterized (Fig. 1). The structure in question has a density of $1.77 \mathrm{~g} \mathrm{~cm}^{-3}$ with $60 \%$ porosity and a modulus of $18 \mathrm{GPa}$. The estimated average pore size is $350 \mu \mathrm{m}$ in diameter by SEM analysis. Larger pores (outer portion of the foam) average $500 \mu \mathrm{m}$ in diameter. The smaller pores (inner portion of the foam) average $200 \mu \mathrm{m}$ in diameter.

\section{Seeding efficiency}

The efficiency of cell seeding in porous surfaces is directly related to the porosity of the material. A higher degree of porosity leads to a more limited surface area in which cells may grow and proliferate. Unlike mesh structures, foams are randomized and do not follow a structural pattern. Therefore, the seeding efficiency of a foam surface is related to the thickness of the sample. A thicker sample will yield a higher seeding efficiency value. A $0.5 \mathrm{~cm}$ thick $60 \%$ porous Ti- $6 \mathrm{Al}-4 \mathrm{~V}$ foam disk yields $\sim 58 \%$ seeding efficiency value.

\section{MTS viability assay}

The evaluation of the viability of cells grown under hypoxia mimetic conditions must be analyzed in order to determine the appropriate amount of stress that the cells

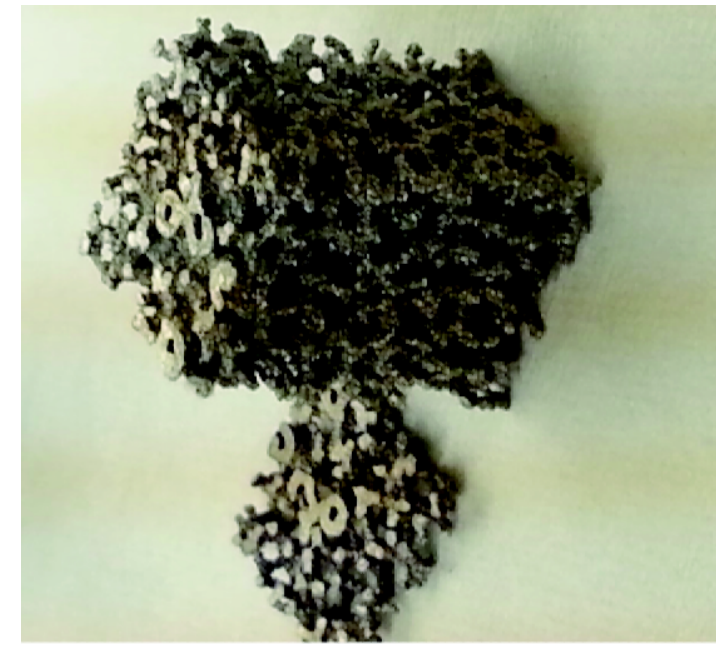

Figure 1 EBM fabricated Ti-6Al-4V foam.

are undergoing, without promoting a cytotoxic effect. The degree of cellular metabolism needs to be assessed, whether cells are grown on a plastic tissue culture plate, a porous metal surface, or a 3D ECM matrix. This experiment serves the purpose of examining the optimal environmental conditions in which cells are stimulated to activate survival signals. Fig. 2 shows a comprehensive MTS viability analysis by varying amount of exposure time and concentration of hypoxia mimetic compounds. The compounds tested include a known toxic hypoxia mimetic $\left(\mathrm{CoCl}_{2}\right)$ and the proposed PHD-2 inhibitor at 1:5 dilutions ranging from $2 \mathrm{mmol} \mathrm{L}^{-1}$ to $640 \mathrm{nmol} \mathrm{L}^{-1}$ of each compound.

As expected, high doses of both compounds decreases cell viability drastically. In most cases, particularly at high concentrations $\mathrm{CoCl}_{2}$ completely eradicated all cells present in the wells (Fig. 2). To the best of our knowledge, the lethal dose at $50 \%$ (LD50) of $\mathrm{CoCl}_{2}$ has not been reported for pre-osteoblast or osteoblast cell populations. Notably, MTS metabolism greatly increases for cells exposed to a hypoxia mimetic condition in periods of time equal or longer than $72 \mathrm{~h}$ for both DFM and $\mathrm{CoCl}_{2}$. This is in accordance to previous reports [31] which show that near $10 \mu \mathrm{mol} \mathrm{L}{ }^{-1}$ DFM can stimulate a survival response. Increased viability at $72 \mathrm{~h}$ of exposure can be attributed to the pre-osteoblast's population doubling time. It is important to mention that, though the goal of this research was to create a hypoxia mimetic environment in the implant, the true levels of oxygen in the environment are not being depleted. Depletion of oxygen de-regulates mitochondrial production of cellular energy. The increased expression of Hif- $1 \alpha$ and VEFG does not ne- 

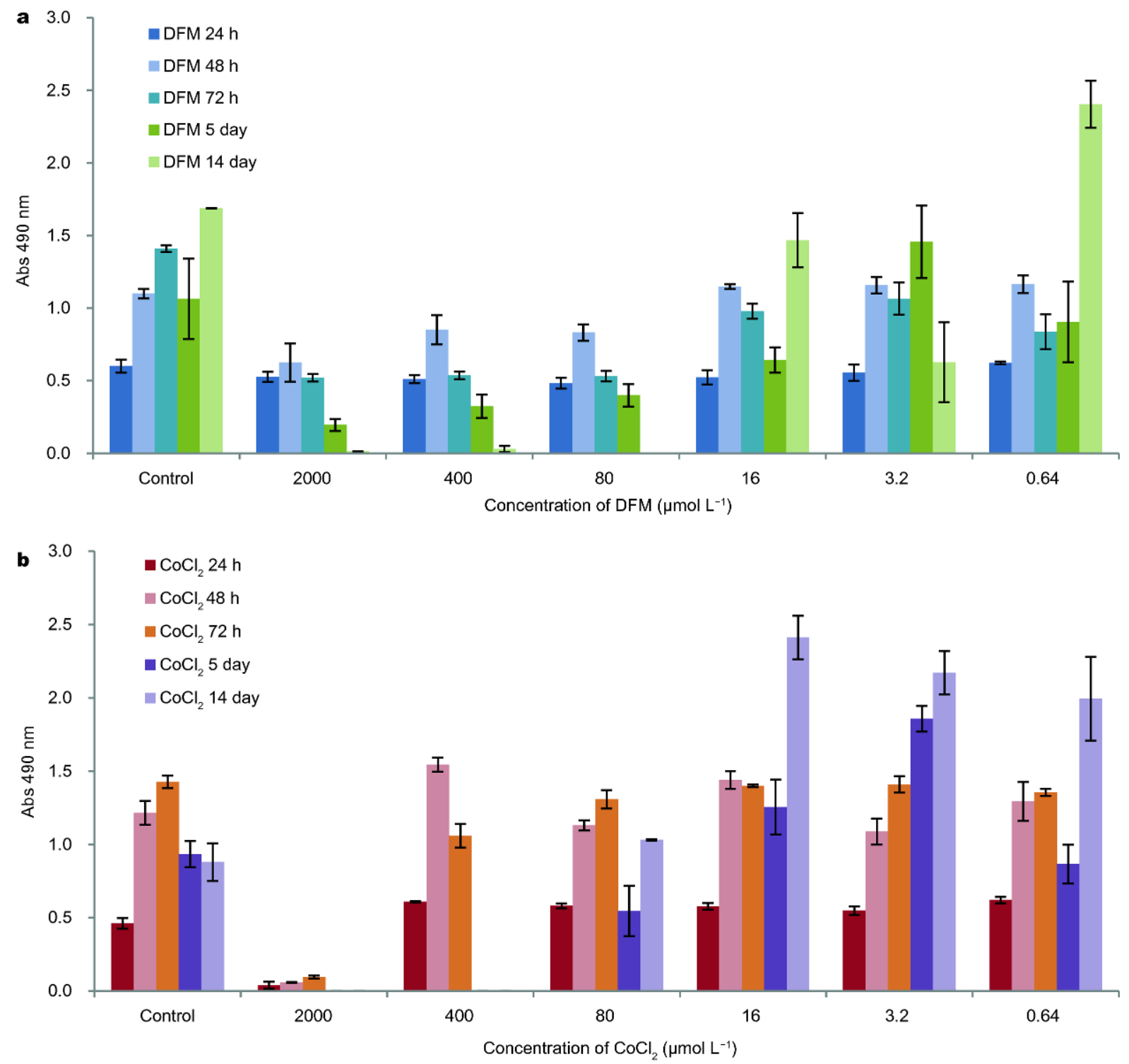

Figure 2 Comprehensive MTS viability assay of MC3T3-E1 pre-osteoblast cells exposed to both $\mathrm{DFM}_{\text {(a) and CoCl }}$ (b).

cessarily result in the de-regulation of mitochondrial energy production processes. Previous reports demonstrate that GST levels do not decrease with increased Hif$1 \alpha$ expression and the uptake of glutamine and glucose significantly increases [23]. Excess glucose is required so that cells may endure the hypoxic reprograming.

\section{Proliferation analysis}

Cell count experiments were performed by trypan blue exclusion dye method for MC3T3-E1 cells (Fig. 3). As expected, a $24 \mathrm{~h}$ exposure to both DFM and $\mathrm{CoCl}_{2}$ dilutions reveals a toxic response to high concentrations of 2 $\mathrm{mmol} \mathrm{L}^{-1}$. Despite seeing no cellular growth in any of the
$2 \mathrm{mmol} \mathrm{L}^{-1} \mathrm{CoCl}_{2}$ wells, there seems to be no immediate apparent loss of cellular proliferation in the remaining wells. The wells containing cells exposed to DFM however, display constant cell population numbers, with a slight decrease in numbers when compared to control cells that were not exposed to any drug at $24 \mathrm{~h}$. The MTS analysis for cells exposed to both DFM and $\mathrm{CoCl}_{2}$ for $24 \mathrm{~h}$ shows almost no variation in cellular metabolism for the different amount of drug concentrations. However, given that the cells exposed to $\mathrm{CoCl}_{2}$ are in fewer numbers and the MTS metabolism remains constant, it could be interpreted that these cells are undergoing stress and may be expressing cellular survival signaling. 

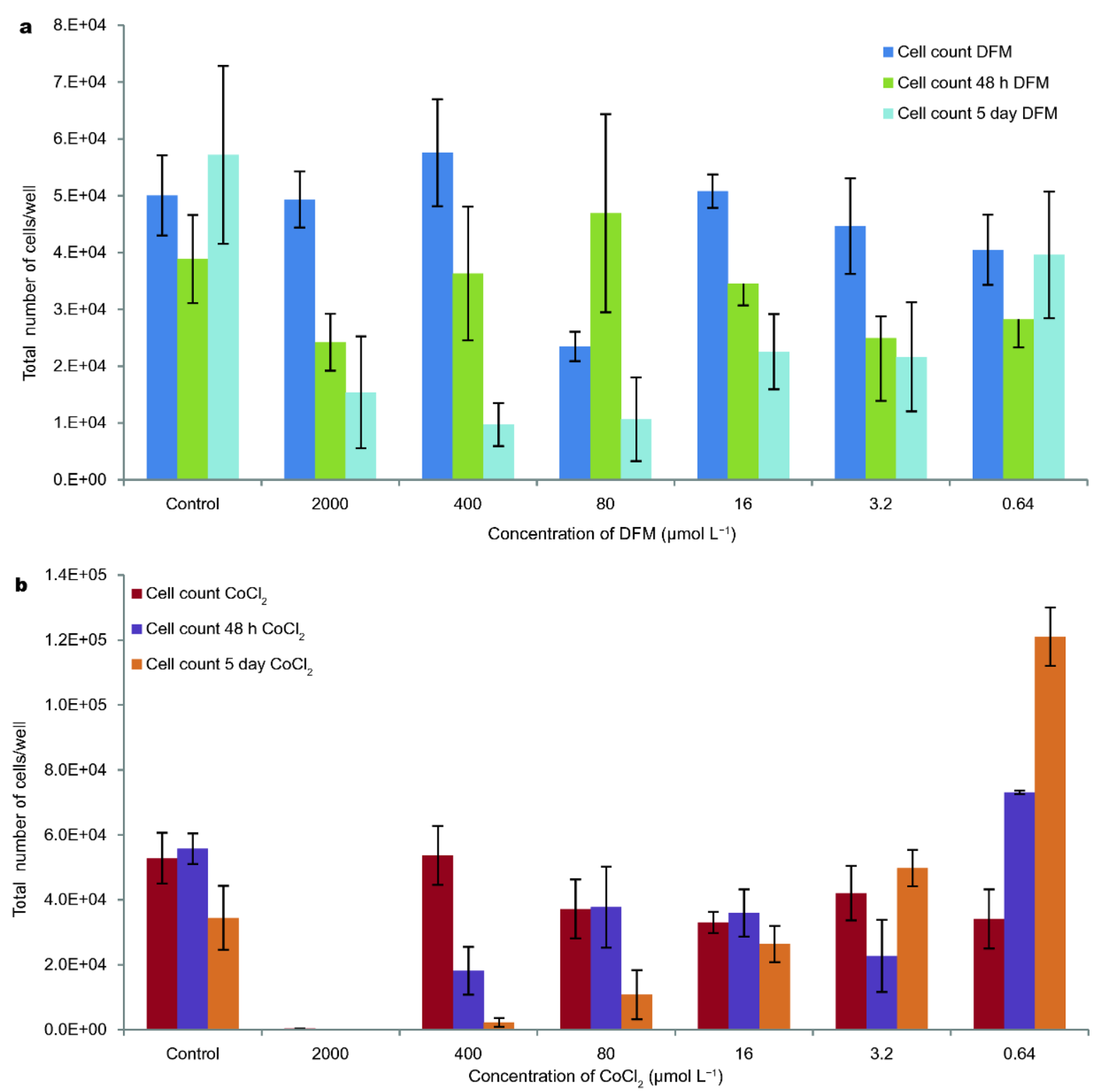

Figure 3 Trypan blue exclusion dye cellular proliferation analysis for MC3T3-E1 cells exposed to DFM (a) and $\mathrm{CoCl}_{2}$ (b) for $24 \mathrm{~h}$.

Given that there was no immediate change in cellular population numbers, but metabolism of MTS readily increases, it can be assumed that cells exposed to both DFM and $\mathrm{CoCl}_{2}$ are expressing survival signaling pathways. More extended time exposures for DFM at high concentrations reveal an obvious decrease in cellular proliferation. This is also as expected, seeing as iron homeostasis is no longer occurring due to DFM saturation. Despite this noticeable decrease, proliferation does not reach untraceable levels, compared to $\mathrm{CoCl}_{2}$ exposed cell populations. An observable increase in proliferation can be especially seen in cells exposed to the lowest concentrations of both DFM and $\mathrm{CoCl}_{2}\left(3.2 \mu \mathrm{mol} \mathrm{L}^{-1}\right.$ and $640 \mathrm{nmol} \mathrm{L}^{-1}$ ). It has been previously reported that HUVECs undergo angiogenesis at approximately $10 \mu \mathrm{mol} \mathrm{L}^{-1}$ DFM [31]. The obtained data suggest that both these cell types can be co-cultured, exposed to low concentrations of DFM $\left(10-1 \mu \mathrm{mol} \mathrm{L}^{-1}\right)$ and achieve a hypoxia mimetic response that can trigger survival signaling pathways.

\section{Fluorescent microscopy}

As previously shown in a research paper by Nune et al. 
[27], MC3T3-E1 cells grow and proliferate in Ti-6Al-4V foams of varying porosity, and a Hoechst 33342 staining in this case yielded similar results (Fig. 4).

Once the appropriate concentration of DFM was determined, MC3T3-E1 cells were seeded and grown in the Ti-6Al-4V foam for 21 day exposed to $3.2 \mu \mathrm{mol} \mathrm{L}^{-1} \mathrm{DFM}$ in a 12 well plate.

\section{Induction of angiogenesis in foams containing hypoxia mimetic ECM}

The capacity to enable the formation of vascular structures is of critical importance for an implant, and therefore a requisite in order to consider the structure as a "living implant". In this experiment, angiogenesis was induced in endothelial cells through exposure to DFM while grown in a 3D collagen based hydrogel. The hydrogel was polymerized in the presence of the printed $\mathrm{Ti}$ $6 \mathrm{Al}-4 \mathrm{~V}$ foam. It has been previously demonstrated that HUVECs exposed to DFM undergo a reorganization process to form capillary in Matrigel $^{\bullet}[31,32]$. The real purpose of this experiment was to analyze whether this process could be interrupted by the presence of metal foams. To achieve this, HUVEC membrane was stained with PKH26 (red fluorescence) whereas MC3T3-E1 cell membrane was stained with PKH67 (green fluorescence).

Once successful staining was confirmed, Ti-6Al-4V foams were seeded with $2 \times 10^{5}$ fluorescently labeled MC3T3-E1 cells and incubated for $12 \mathrm{~h}$ to allow cellular adherence. After expiration of this time, Matrigel ${ }^{\circledR}$ was added to the foam containing the pre-incubated MC3T3E1 cells. After an $18 \mathrm{~h}$ period, HUVECs that were exposed to $2 \mathrm{mmol} \mathrm{L}^{-1}$ DFM readily formed capillaries (Fig. 5), suggesting the success of the approach. In the control setting (no DFM), these capillaries were not apparent (Fig. 5). The integrity of the capillaries could not be measured. However, the capillaries seem to maintain their structure for an extended period of time. This can be measured because the cells used in this assay fluoresce without having to fix cells or stain with toxic dyes.

The branching of these structures is shown. As previously mentioned, the experimental setting were $\mathrm{HU}$ VECs treated with $2 \mathrm{mmol} \mathrm{L}^{-1} \mathrm{DFM}$ at the moment of cellular resuspension.

Fluorescent microscopy becomes challenging when analyzing 3D cell cultures, and solid foams add complexity to the analysis. Fig. 6 demonstrates that vascular structures may form in foams with an ECM-like hydrogel matrix. This result is central to the demonstration that vascularization is achievable in these materials, and supports the hypothesis that a foam does not present a

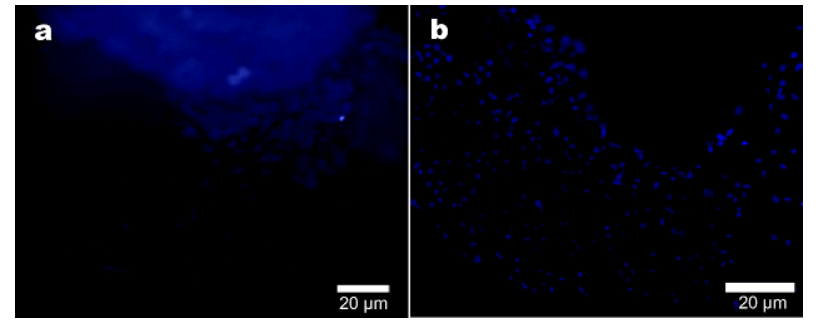

Figure 4 MC3T3 cells grown for 21 day without DFM (a), and MC3T3 cells grown for 21 day exposed to $3.2 \mu \mathrm{mol} \mathrm{L} \mathrm{L}^{-1} \mathrm{DFM}$ (b).

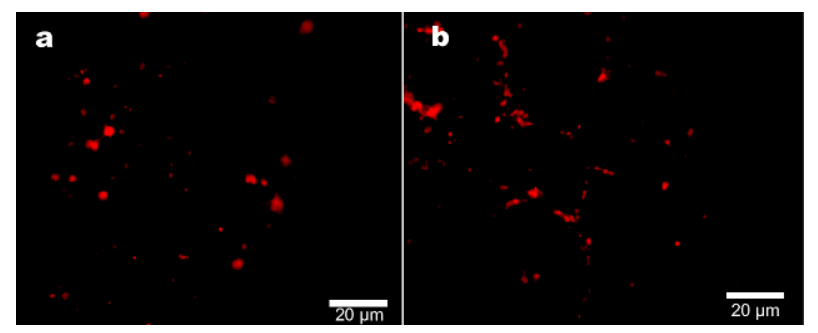

Figure 5 HUVECs with no DFM treatment in Matrigel (a), and HUVECs with $2 \mathrm{mmol} \mathrm{L}^{-1}$ DFM treatment after $18 \mathrm{~h}$ (Day 1 ).

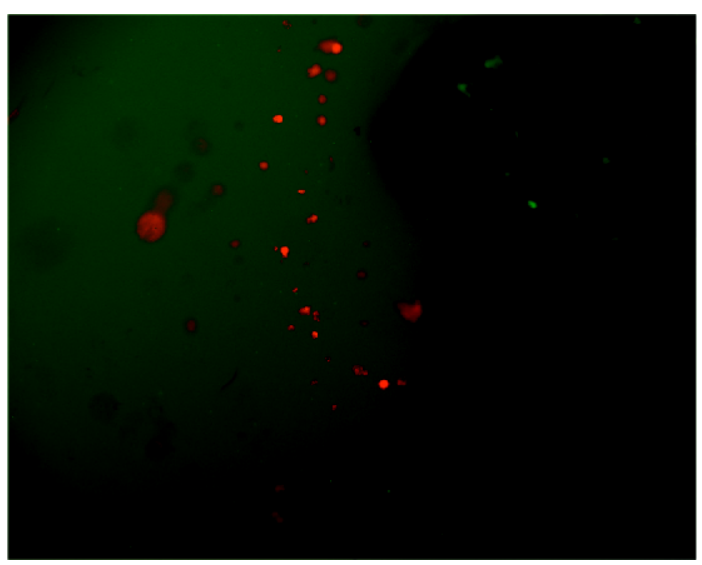

Figure 6 Vascular network formation on foams and Matrigel mix under DFM treatment. HUVECs (red) \& MC3T3-E1 (green).

physical barrier that hinders this biological process. Further optimizations are required however, to establish the occurrence of this process in greater detail and resolution.

\section{Cell morphology and adhesion}

The distribution of cellular growth on the implant was analyzed by SEM imaging. SEM provides the advantage of analyzing the porous surface in which the pre-osteoblasts grow. It has been previously shown that MC3T3-E1 cells undergo cell differentiation when grown in foams of various densities under pre-osteoblast differentiation 


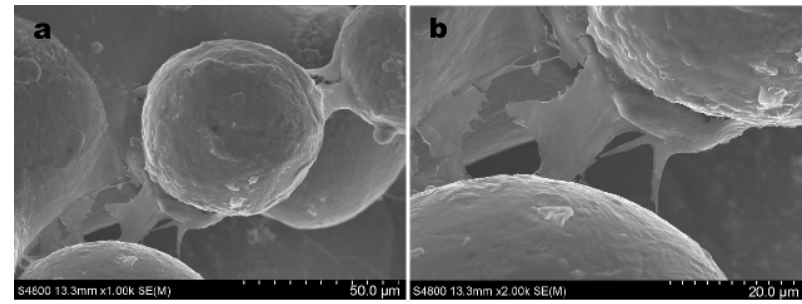

Figure 7 SEM imaging of MC3T3-E1 cells grown for 21 day in a $60 \%$ porous $\mathrm{Ti}-6 \mathrm{Al}-4 \mathrm{~V}$ foam in non-differentiation media.

media (a-MEM 10\% FBS + glutamine) [28]. Fig. 7 shows MC3T3-E1 cells grown for 21 day in non-differentiation media on a $60 \%$ porous Ti- $6 \mathrm{Al}-4 \mathrm{~V}$ foam.

An SEM analysis was made in the same foam (Fig. 7), but under a hypoxic mimetic environment with DFM. As previously shown, MC3T3-E1 cells undergo a drastic morphological change under the presence of DFM. The cells display an "elongation" which imparts on them a fibroblastic morphology.

\section{Alizarin red $\mathrm{S}$ mineralization assay}

It has been previously reported that MC3T3-E1 cells undergo cellular differentiation into osteoblasts in foams and other Ti-6Al-4V surfaces, and that they are able to mineralize calcium when grown in these surfaces [28]. Under ARS staining, the calcium nodes synthesized by the cells are apparent, particularly for lower DFM concentrations (Fig. 8). The cells underwent evident morphological changes, as previously seen under DFM exposures (Fig. 5).

The synthesis of hydroxyapatite can be interpreted as a sign of pre-osteoblast differentiation given that the cell's phenotype expresses forms of mineralized calcium nodes. Despite seeing calcium nodes as early as 7 day (Fig. 9b), the production of hydroxyapatite is small in quantity, which correlates with previous reports [28]. However, synthesis of this main component of bone structure is mostly observed at lower concentrations of DFM. Not only are the cells proliferating, but differentiating to a phenotype that promotes structural bone component formation. At 14 day of exposure to DFM the calcium nodes appear more obvious in all wells when compared to a 7 day exposure. In all wells the levels of mineralized calcium seem apparent but they are most notable in the lower DFM concentration exposures. The highest expression of mineralized calcium was observed at the 640 nmol L ${ }^{-1}$ DFM exposed cells for 21 day (Fig. 9d). Notably, in every instance, the expression of hydroxyapatite is always in greater amount for the cells exposed to 640 nmol L $\mathrm{L}^{-1}$ DFM when compared to control cells.

It has been previously shown that MC3T3-E1 cells undergo cellular differentiation and successfully synthesize hydroxyapatite when grown on 3D printed Ti-6Al$4 \mathrm{~V}$ foams [28]. Here, it is demonstrated that cellular production of hydroxyapatite can be enhanced by addition of $3.2 \mu \mathrm{mol} \mathrm{L} \mathrm{L}^{-1} \mathrm{DFM}$.

Initially, there is a definitive amount of hydroxyapatite being synthesized by the osteoblasts in both cases. Cells exposed to DFM express comparatively similar amounts

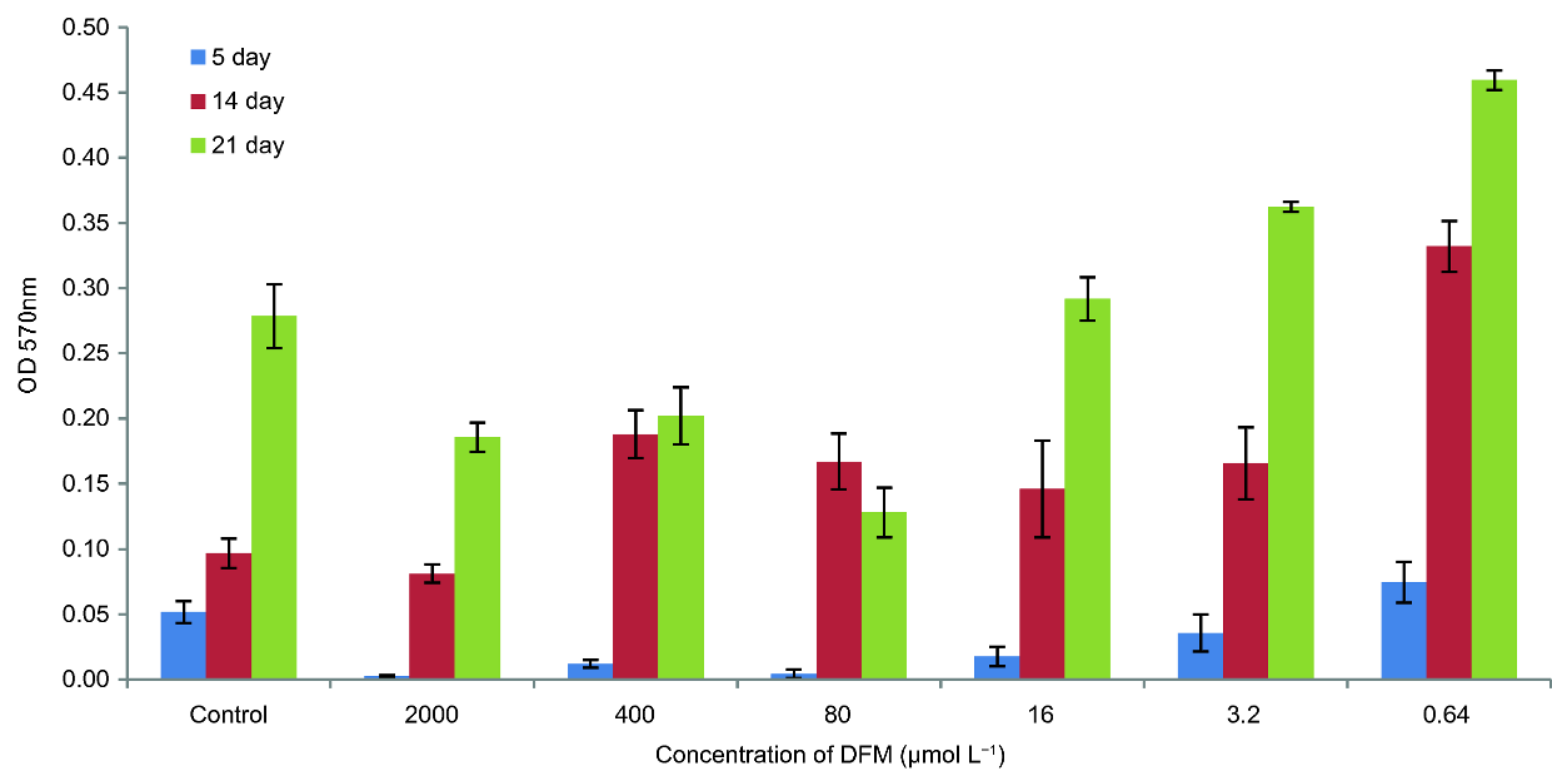

Figure 8 Alizarin red S staining assay performed on MC3T3-E1 cells exposed to various concentrations of DFM. 


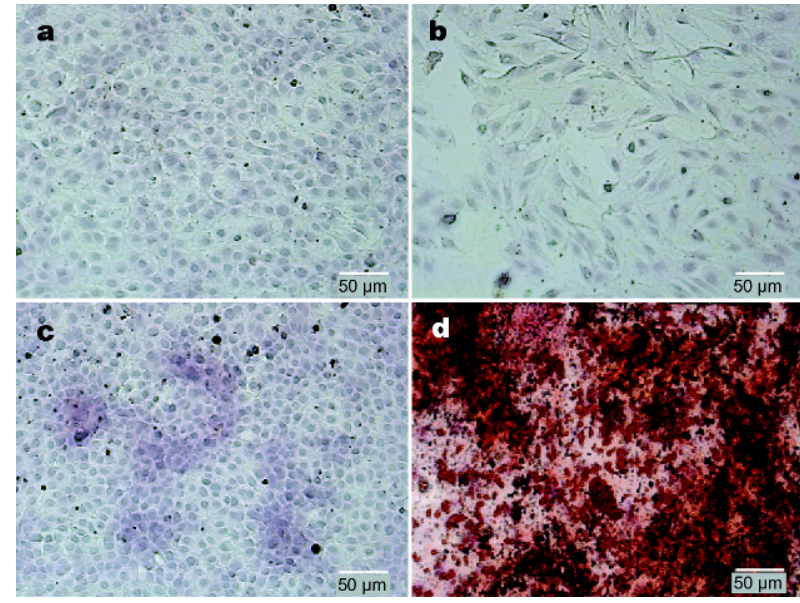

Figure 9 Alizarin red S staining assay of MC3T3-E1 cells exposed to various DFM concentrations. (a) Control cells not exposed to DFM, (b) cells exposed to $640 \mathrm{nmol} \mathrm{L}{ }^{-1} \mathrm{DFM}$ for 7 day, (c) cells exposed to 2 mmol L ${ }^{-1}$ DFM for 7 day, and (d) cells exposed to $640 \mathrm{nmol} \mathrm{L}^{-1} \mathrm{DFM}$ for 21 day.

of hydroxyapatite to control cells. Regardless of the hypoxic mimetic stress, where cells may be metabolically challenged to survive, the synthesis of hydroxyapatite does not seem to be suppressed. This can also be observed at longer time exposures of 14 day, the synthesis levels of hydroxyapatite seem to increase on par in both settings. After 21 day of exposure, the amount of cellular hydroxyapatite dramatically increases on the foam with cells exposed to DFM (Fig. 10). The foam containing cells exposed to $3.2 \mu \mathrm{mol} \mathrm{L} \mathrm{L}^{-1}$ DFM displays a regular coating of hydroxyapatite (orange), and in considerably larger amounts than the control grown without DFM (Figs 10 and 11).

\section{DISCUSSION}

This research was conducted to examine the possibility of successfully achieving vascular structure formation in additive manufactured Ti-6Al-4V foams in order to design a fully living implant. The results show that this proposed approach can be utilized as a potential prototype for bone replacement implants. Foams provide an ideal substrate to substitute bone due to their random distribution and interconnection, which is largely similar to that of real bone. Ti-6Al-4V has been a popular alloy used in the biomedical industry and research and has been extensively characterized [33-36]. The limitation of free iron availability through exposure of DFM seems to be a driving factor to enhance the synthesis of hydroxyapatite by cells. It has been previously demonstrated that pre-osteoblasts proliferate, differentiate and are able

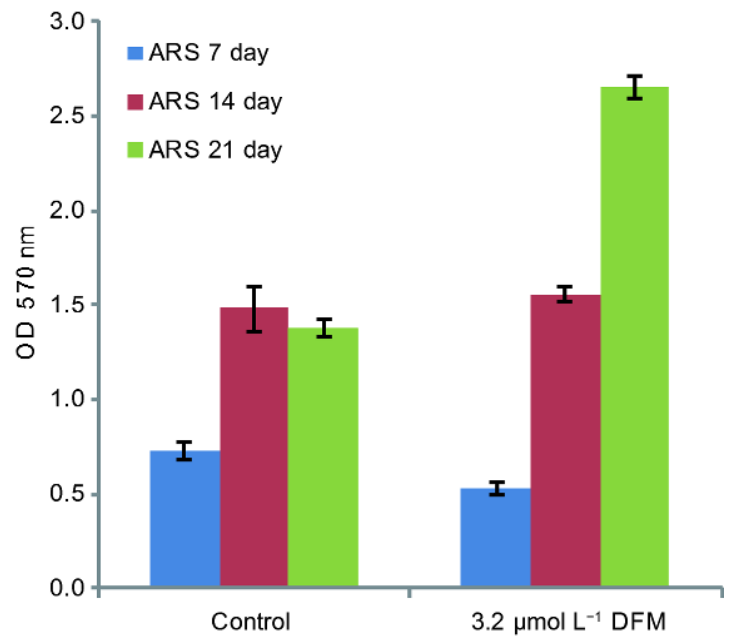

Figure 10 ARS analysis performed on MC3T3-E1 cells grown in foams for 7, 14 and 21 day. Negative controls represent cells without DFM exposure. Experimental setting represents cell exposed to $3.2 \mu \mathrm{mol} \mathrm{L}$ DFM.
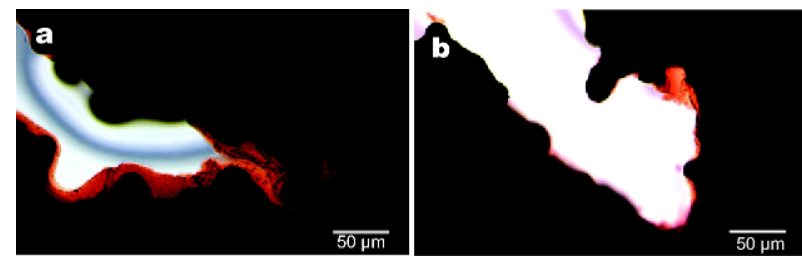

Figure 11 ARS staining on foams after 21 day MC3T3-E1 incubation with no DFM (a) and with $3.2 \mu \mathrm{mol} \mathrm{L}^{-1}$ DFM (b).

to synthesize hydroxyapatite when grown on foam and mesh structures of this alloy. However, this is the first time that the formation of a vascular network formation has been achieved in the presence of this foam alloy. The process of vascular structure initiation has a key step that involves proteolytic degradation of the ECM so that endothelial cells can migrate to form the microcapillary [31]. DFM has proven to be a suitable candidate molecule to promote vascularization of endothelial cells. Immediate biomedical applications of this iron chelating agent are viable, seeing as it is already been FDA approved for the treatment of iron poisoning. This research has greatly focused on the process of forming a vascular network. However crucial this step may be, the concept of a living implant pertains to various other cellular molecular mechanisms, mainly involved in wound healing and the regeneration of tissue. Tissue that has undergone extensive damage needs to endure harsh environments that stimulate apoptosis rather than cell survival. A tissuesolid metal interface is not sufficient to promote a wound healing process, the ingrowth of bone, and eventually the 
formation of a vascular network. Implanted solid metal bars present a physical limitation to the availability of nutrients and, most notably, oxygen. The wounded tissue then suffers from hypoxia, triggering an irreversible response that eventually leads to cellular death. In a heavily wounded tissue metabolic demands differ vastly from that of normal tissue. To create a fully living implant that mimics real tissue, this issue needs to be addressed and thoroughly understood. Therefore, the addition of molecules that can compensate for the metabolic high demands is required. It is to this end that $\mathrm{D}(+)$ glucose was added to cells undergoing a hypoxia mimetic response. The addition of glutamine to enhance cell survival is also suggested by Stegen et al. [22] in order to reduce the production of ROS through glutathione, which is a powerful biological reducing agent.

The materials chosen and tested in this research prove to be a combination that is suitable to develop a fully living bone replacement implant. Ti-6Al-4V foams provide structural support, while an ECM-like hydrogel simulates an aqueous microenvironment that drives wound healing, bone ingrowth and vascularization. Despite the attractive properties of Matrigel, this product is not intended for anything other than research purposes. However, its main constituents may be further utilized with the focus of creating a hydrogel capable of driving the before mentioned processes. Collagen and gelatin hydrogels can be tailored to maintain their solid stability under physiological conditions $[4,37]$. Despite the success of achieving vascularization in the presence of Ti-6Al-4V foams, in vivo implantation of this mixture of materials is required for a more comprehensive analysis of the ingrowth of bone and the ability of vascular networks to form. The effects of mechanical loading on the hydrogel matrix in Ti-6Al-4V foams are in need of evaluation $[38,39]$. There is need of further analysis of the metabolic demands of stressed tissues, mainly in areas where the availability of oxygen is limited. Another approach to optimize the delivery of DFM in hydrogels could be the microencapsulation of DFM and adding the microcapsules as a gradient in the gels [40]. This may enable the specific driving of tissue ingrowth into the implant. This approach could also be applied to siRNAs that silence PHD-2 activity [23]. Utilizing siRNAs could be a more suitable approach due to its specificity. Iron chelation is not specific as to the processes that may be altered. Iron (III) is a necessary supplement as it is used as a cofactor to various enzymes, and it is also necessary for electron transport in aerobic metabolism. However, with respect to bone tissue repair, the limitation of iron (III) availability decreases bone resorption. This suggests that this limitation should enhance the ingrowth of bone but it may also affect other unrelated cellular processes.

This research proves that additive manufactured Ti$6 \mathrm{Al}-4 \mathrm{~V}$ foams do not present a physical barrier, nor do they inhibit the formation of an endothelium network under physiological conditions. The foam itself is not able to drive this process, but the combination of materials and effecting molecules is sufficient to cause its desired effect. Soluble DFM in a hydrogel matrix demonstrated to help drive angiogenesis and enhance the synthesis of hydroxyapatite.

\section{CONCLUSIONS}

This work demonstrates that a mixture of additive manufactured Ti-6Al-4V foam in combination with a collagen based hydrogel matrix containing DFM, a hypoxia mimetic compound, can form vasculature under physiological conditions, while maintaining osteoblast cell differentiation and proliferation. This approach induces a hypoxia mimetic stress that will trigger cellular survival signals that ultimately enhances wound healing processes in bone.

The conclusions from this research are summarized as follows: (1) Additive manufactured Ti-6Al-4V foams in combination with Matrigel infused with DFM allows vascular network formation. (2) DFM improves pre-osteoblast differentiation and synthesis of hydroxyapatite both in tissue culture plates and in foams. (3) Vascular networks maintain their stability in gels for extended periods of time in matrigel. (4) Foams prove to be a suitable material to allow the ingrowth of bone. Further research is required to understand and meet the metabolic demands of damaged/stressed tissue to promote the ingrowth of bone.

\section{Received 25 May 2017; accepted 4 August 2017;} published online 10 October 2017

1 Kang HW, Lee SJ, Ko IK, et al. A 3D bioprinting system to produce human-scale tissue constructs with structural integrity. Nat Biotechnol, 2016, 34: 312-319

2 Chung IM, Enemchukwu NO, Khaja SD, et al. Bioadhesive hydrogel microenvironments to modulate epithelial morphogenesis. Biomaterials, 2008, 29: 2637-2645

3 Nicodemus GD, Bryant SJ. Cell encapsulation in biodegradable hydrogels for tissue engineering applications. Tissue Eng Part BRev, 2008, 14: 149-165

4 Nguyen LH, Annabi N, Nikkhah M, et al. Vascularized bone tissue engineering: approaches for potential improvement. Tissue Eng Part B-Rev, 2012, 18: 363-382

5 Bertassoni LE, Cecconi M, Manoharan V, et al. Hydrogel bioprinted microchannel networks for vascularization of tissue en- 
gineering constructs. Lab Chip, 2014, 14: 2202-2211

6 Zhang X, Yan X, Cheng L, et al. Wound healing improvement with PHD-2 silenced fibroblasts in diabetic mice. PLoS ONE, 2013, 8: e84548

7 Samelko L, Caicedo MS, Lim SJ, et al. Cobalt-alloy implant debris induce HIF-1a hypoxia associated responses: a mechanism for metal-specific orthopedic implant failure. PLoS ONE, 2013, 8: e67127

8 Unger RE, Dohle E, Kirkpatrick CJ. Improving vascularization of engineered bone through the generation of pro-angiogenic effects in co-culture systems. Adv Drug Deliver Rev, 2015, 94: 116-125

9 Jung HD, Jang TS, Wang L, et al. Novel strategy for mechanically tunable and bioactive metal implants. Biomaterials, 2015, 37: 49-61

10 Sobral JM, Caridade SG, Sousa RA, et al. Three-dimensional plotted scaffolds with controlled pore size gradients: effect of scaffold geometry on mechanical performance and cell seeding efficiency. Acta Biomater, 2011, 7: 1009-1018

11 Phelps EA, Headen DM, Taylor WR, et al. Vasculogenic bio-synthetic hydrogel for enhancement of pancreatic islet engraftment and function in type 1 diabetes. Biomaterials, 2013, 34: 4602-4611

12 Kusumbe AP, Ramasamy SK, Adams RH. Coupling of angiogenesis and osteogenesis by a specific vessel subtype in bone. Nature, 2014, 507: 323-328

13 Novosel EC, Kleinhans C, Kluger PJ. Vascularization is the key challenge in tissue engineering. Adv Drug Deliver Rev, 2011, 63: 300-311

14 Snyder CM, Chandel NS. Mitochondrial regulation of cell survival and death during low-oxygen conditions. Antioxidants Redox Signal, 2009, 11: 2673-2683

15 Alvarez-Tejado M, Naranjo-Suarez S, Jiménez C, et al. Hypoxia induces the activation of the phosphatidylinositol 3-Kinase/Akt cell survival pathway in PC12 cells. J Biol Chem, 2001, 276: 2236822374

16 Chen LL, Huang M, Tan JY, et al. PI3K/AKT pathway involvement in the osteogenic effects of osteoclast culture supernatants on preosteoblast cells. Tissue Eng Part A, 2013, 19: 2226-2232

17 Liu $\mathrm{P}, \mathrm{He} \mathrm{K}$, Song $\mathrm{H}$, et al. Deferoxamine-induced increase in the intracellular iron levels in highly aggressive breast cancer cells leads to increased cell migration by enhancing TNF- $\alpha$-dependent NF- $\kappa \mathrm{B}$ signaling and TGF- $\beta$ signaling. J Inorg Biochem, 2016, 160: 40-48

18 Zhang Y, Zhao X, Chang Y, et al. Calcium channel blockers ameliorate iron overload-associated hepatic fibrosis by altering iron transport and stellate cell apoptosis. Toxicol Appl Pharmacol, 2016, 301: 50-60

19 Xie W, Lorenz S, Dolder S, et al. Extracellular iron is a modulator of the differentiation of osteoclast lineage cells. Calcif Tissue Int, 2016, 98: 275-283

20 Kontoghiorghe CN, Kontoghiorghes GJ. Efficacy and safety of iron-chelation therapy with deferoxamine, deferiprone, and deferasirox for the treatment of iron-loaded patients with nontransfusion-dependent thalassemia syndromes. Drug Des Devel Ther, 2016, 10: 465-481

21 Guo JP, Pan JX, Xiong L, et al. Iron chelation inhibits osteoclastic differentiation in vitro and in Tg2576 mouse model of Alzheimer's disease. PLoS ONE, 2015, 10: e0139395

22 Stegen S, van Gastel N, Eelen G, et al. HIF-1a promotes glutaminemediated redox homeostasis and glycogen-dependent bioenergetics to support postimplantation bone cell survival. Cell Metabolism, 2016, 23: 265-279

23 Selvaraju V, Parinandi NL, Adluri RS, et al. Molecular mechanisms of action and therapeutic uses of pharmacological inhibitors of HIF-prolyl 4-hydroxylases for treatment of ischemic diseases. Antioxidants Redox Signal, 2014, 20: 2631-2665

24 Jain IH, Zazzeron L, Goli R, et al. Hypoxia as a therapy for mitochondrial disease. Science, 2016, 352: 54-61

25 Karuppagounder SS, Alim I, Khim SJ, et al. Therapeutic targeting of oxygen-sensing prolyl hydroxylases abrogates ATF4-dependent neuronal death and improves outcomes after brain hemorrhage in several rodent models. Sci Translational Med, 2016, 8: 328ra29328 ra29

26 Sugiyama T, Uo M, Wada $\mathrm{T}$, et al. Detection of trace metallic elements in oral lichenoid contact lesions using SR-XRF, PIXE and XAFS. Sci Rep, 2015, 5: 10672

27 Nune KC, Misra RDK, Gaytan SM, et al. Interplay between cellular activity and three-dimensional scaffold-cell constructs with different foam structure processed by electron beam melting. J Biomed Mater Res, 2015, 103: 1677-1692

28 Li D, Liu J, Guo B, et al. Osteoclast-derived exosomal miR-214-3p inhibits osteoblastic bone formation. Nat Commun, 2016, 7: 10872

29 Li SJ, Xu QS, Wang Z, et al. Influence of cell shape on mechanical properties of $\mathrm{Ti}-6 \mathrm{Al}-4 \mathrm{~V}$ meshes fabricated by electron beam melting method. Acta Biomater, 2014, 10: 4537-4547

30 Veschini L, Belloni D, Foglieni C, et al. Hypoxia-inducible transcription factor-1 alpha determines sensitivity of endothelial cells to the proteosome inhibitor bortezomib. Blood, 2007, 109: 25652570

31 Donneys A, Weiss DM, Deshpande SS, et al. Localized deferoxamine injection augments vascularity and improves bony union in pathologic fracture healing after radiotherapy. Bone, 2013, 52: 318325

32 Gaytan SM, Murr LE, Martinez E, et al. Comparison of microstructures and mechanical properties for solid and mesh cobaltbase alloy prototypes fabricated by electron beam melting. Metall Mat Trans A, 2010, 41: 3216-3227

33 Murr LE, Quinones SA, Gaytan SM, et al. Microstructure and mechanical behavior of $\mathrm{Ti}-6 \mathrm{Al}-4 \mathrm{~V}$ produced by rapid-layer manufacturing, for biomedical applications. J Mech Behav Biomed Mater, 2009, 2: 20-32

34 Murr LE, Amato KN, Li SJ, et al. Microstructure and mechanical properties of open-cellular biomaterials prototypes for total knee replacement implants fabricated by electron beam melting. J Mech Behav Biomed Mater, 2011, 4: 1396-1411

35 Murr LE, Gaytan SM, Martinez E, et al. Next generation orthopaedic implants by additive manufacturing using electron beam melting. Int J Biomaterials, 2012, 2012: 1-14

36 Bertassoni LE, Cardoso JC, Manoharan V, et al. Direct-write bioprinting of cell-laden methacrylated gelatin hydrogels. Biofabrication, 2014, 6: 024105

37 Rivron NC, Vrij EJ, Rouwkema J, et al. Tissue deformation spatially modulates VEGF signaling and angiogenesis. Proc Natl Acad Sci USA, 2012, 109: 6886-6891

38 Klein-Nulend J, Bacabac R, Bakker A. Mechanical loading and how it affects bone cells: the role of the osteocyte cytoskeleton in maintaining our skeleton. eCM, 2012, 24: 278-291

39 Rassu G, Salis A, Porcu EP, et al. Composite chitosan/alginate hydrogel for controlled release of deferoxamine: a system to potentially treat iron dysregulation diseases. Carbohydrate Polymers, 2016, 136: 1338-1347

40 Lee $\mathrm{BH}, \mathrm{Li} \mathrm{B}$, Guelcher SA. Gel microstructure regulates proliferation and differentiation of MC3T3-E1 cells encapsulated in 
alginate beads. Acta Biomater, 2012, 8: 1693-1702

Acknowledgements We thank Dr. Sara Gaytan for her assistance with the fabrication of the Ti-6Al-4V foams and their characterization. We thank Dr. Robert Kirken and Dr. Georgialina Rodríguez for their support and assistance with the western blot analysis and the purchasing of antibodies, and Nadia Rocha for her assistance. We thank Dr. Armando Varela for his support and assistance with the fluorescent microscopy experiments and his insights for some experimental designs and Gladys Almodóvar for her assistance with cell culture techniques and for providing equipment necessary for the completion of this work. Finally, we are grateful for the support of this work through a research assistantship to VCR made possible by Dr. Roberto Osegueda through the Office of Research and Special Projects.

Author contributions Correa VL performed all of the experiments in this research. Garza KM coordinated and designed the cell proliferation assay and the $\mathrm{PKH}$ dye staining for the angiogenesis assay. Murr LE conceived and supervised the project.

Conflict of interest The authors declare that they have no conflict of interest.

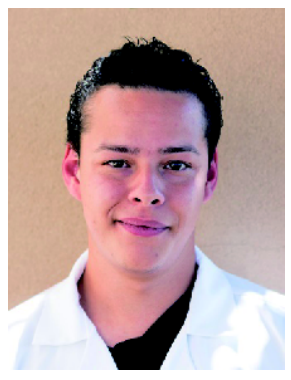

Victor L. Correa is a post-doctoral fellow in the laboratory of Dr. Laura O'Dell in the Department of Psychology at the University of Texas at El Paso. He holds a BSc degree in Cell \& Molecular Biology from the Universidad Metropolitana in San Juan, Puerto Rico, a MSc degree in Metallurgical \& Materials Science Engineering, and a PhD in Materials Science Engineering from the University of Texas at El Paso. His current research interests integrate his background in engineering and biology to develop novel models of tissue regeneration and cellular adaptation, with a current focus on the effects of drugs of abuse on the clinical efficacy of these models. 